\title{
Local Dynamical Instabilities in Magnetized, Radiation Pressure Supported Accretion Disks
}

\author{
Omer Blaes and Aristotle Socrates \\ Department of Physics, University of California, Santa Barbara, CA 93106
}

\begin{abstract}
We present a general linear dispersion relation which describes the coupled behavior of magnetorotational, photon bubble, and convective instabilities in weakly magnetized, differentially rotating accretion disks. We presume the accretion disks to be geometrically thin and supported vertically by radiation pressure. We fully incorporate the effects of a nonzero radiative diffusion length on the linear modes. In an equilibrium with purely vertical magnetic field, the vertical magnetorotational modes are completely unaffected by compressibility, stratification, and radiative diffusion. However, in the presence of azimuthal fields, which are expected in differentially rotating flows, the growth rate of all magnetorotational modes can be reduced substantially below the orbital frequency. This occurs if diffusion destroys radiation sound waves on the length scale of the instability, and the magnetic energy density of the azimuthal component exceeds the non-radiative thermal energy density. While sluggish in this case, the magnetorotational instability still persists and will still tap the free energy of the differential rotation. Photon bubble instabilities are generically present in radiation pressure dominated flows where diffusion is present. We show that their growth rates are limited to a maximum value which is reached at short wavelengths where the modes may be viewed as unstable slow magnetosonic waves. We also find that vertical radiation pressure destabilizes upward propagating fast waves, and that Alfvén waves can be unstable. These instabilities typically have smaller growth rates than the photon bubble/slow modes. We discuss how all these modes behave in various regimes of interest, and speculate how they may affect the dynamics of real accretion disk flows.
\end{abstract}

Subject headings: accretion, accretion disks — black hole physics — instabilities - MHD 


\section{Introduction}

The physical state of the radiation pressure dominated, innermost regions of accretion disks around black holes has been uncertain ever since the early days of accretion disk theory. Standard alpha disk models in which the viscous stress is assumed to scale with the radiation pressure are subject to thermal and viscous instabilities (Lightman \& Eardley 1974, Shakura \& Sunyaev 1976). These instabilities are sensitive to the assumed prescription for the anomalous viscosity (e.g. Piran 1978), so it remains unclear whether or not they are actually present in real flows. If they are, the accretion flow may adopt a radically different state from that usually envisaged in thin accretion disk theory, such as the multiphase equilibrium recently proposed by Krolik (1998).

In addition to these secular instabilities, dynamical instabilities also exist. First and foremost, a differentially rotating flow with a negative angular velocity gradient and an initially weak magnetic field is unstable to the magnetorotational instability (MRI, Balbus \& Hawley 1991). The turbulence resulting from this instability is currently the most plausible mechanism known for generating the anomalous viscosity required in accretion disk models. Dissipation of magnetohydrodynamic waves excited by this turbulence by photon diffusion and photon viscosity has recently been examined by Agol \& Krolik (1998). Photon diffusion might also affect the linear development of the instability itself in a laminar, radiation pressure dominated flow, but this issue has not been examined previously.

Gammie (1998) has suggested that the overstable photon bubble modes discussed by Arons (1992) in the context of X-ray pulsars also exist in radiation dominated accretion flows in general. However, his instability analysis was limited to studying a static equilibrium where the effects of differential rotation were entirely neglected. Pietrini \& Krolik (2000) have recently investigated convective instabilities in unmagnetized, radiation pressure dominated, differentially rotating flows. They assumed a constant vertical density profile, which necessarily leads to an unstable entropy gradient. Whether or not such unstable gradients exist in reality depends on the vertical dissipation profile, which in turn depends on the structure of the MHD turbulence.

These dynamical instabilities may all play a role at some level in the dynamics and thermodynamics of the radiation pressure dominated portion of accretion disks. It is the purpose of this paper to provide a unified description of all three by deriving a general linear dispersion relation which incorporates them all. Such an analysis will hopefully prove to be a useful guide to numerical simulations which explore the nonlinear development of these instabilities and their effects on the resulting turbulent state of the inner accretion disk.

This paper is organized as follows. In section 2 we discuss our basic equations and 
assumptions. Then in section 3 we focus exclusively on the MRI by deriving its dispersion relation in the absence of vertical stratification so that the other instabilities are suppressed. We are therefore able to study just the effects of photon diffusion on what is probably the most important of these instabilities. In section 4 we then present the full dispersion relation for a vertically stratified, differentially rotating medium, and discuss its solutions. We discuss the relevance of these solutions to astrophysical accretion disks in section 5 and then summarize our conclusions in section 6 .

\section{Equations and Assumptions}

In Eulerian coordinates in an inertial frame, the equations of radiation MHD which we use in this paper are

$$
\begin{gathered}
\frac{\partial \rho}{\partial t}+\boldsymbol{\nabla} \cdot(\rho \mathbf{v})=0 \\
\rho\left(\frac{\partial \mathbf{v}}{\partial t}+\mathbf{v} \cdot \boldsymbol{\nabla} \mathbf{v}\right)=-\nabla p-\rho \boldsymbol{\nabla} \Phi+\frac{1}{4 \pi}(\nabla \times \mathbf{B}) \times \mathbf{B}+\frac{\kappa_{\mathrm{es}} \rho}{c} \mathbf{F} \\
\frac{\partial p}{\partial t}+\mathbf{v} \cdot \boldsymbol{\nabla} p=\frac{\gamma p}{\rho}\left(\frac{\partial \rho}{\partial t}+\mathbf{v} \cdot \nabla \rho\right) \\
\frac{\partial E}{\partial t}+\mathbf{v} \cdot \boldsymbol{\nabla} E+\frac{4}{3} E \boldsymbol{\nabla} \cdot \mathbf{v}=-\boldsymbol{\nabla} \cdot \mathbf{F} \\
\mathbf{F}=-\frac{c}{3 \kappa_{\mathrm{es}} \rho} \nabla E
\end{gathered}
$$

and

$$
\frac{\partial \mathbf{B}}{\partial t}=\nabla \times(\mathbf{v} \times \mathbf{B}) .
$$

Here $\rho$ is the fluid mass density, $\mathbf{v}$ is the velocity, $p$ is the gas pressure, $\mathbf{B}$ is the magnetic field, $\gamma$ is the gas adiabatic index, $E$ is the radiation energy density, $\mathbf{F}$ is the radiative flux, and $c$ is the speed of light. We have followed the notation of Stone, Mihalas, \& Norman (1992) with regard to variables associated with the radiation field. In order to facilitate comparison with the work of Gammie (1998), we note that his $p_{g}$ is our $p$, his $J$ is $c E / 4 \pi$, and his $\mathbf{H}$ is $\mathbf{F} / 4 \pi$.

We neglect the self-gravity of the fluid, and assume that the gravitational potential $\Phi$ originates from the central mass. We completely neglect relativistic effects. We also assume a pure electron scattering opacity $\kappa_{\mathrm{es}}$, neglecting absorption opacity entirely. This should be a good first approximation to the inner parts of accretion disks around black holes which are electron scattering dominated. Note, however, that this has the consequence that the gas and radiation are coupled together purely by momentum exchange. They do not 
exchange heat, which is why the gas energy equation (3) simply reduces to the condition for adiabatic flow. This approximation still recovers the basic MRI and photon bubble instabilities, and so we believe captures the physics that we wish to explore here.

We have closed the radiation moment equations by assuming that the radiation field is close to isotropic, so that the stress tensor is diagonal with elements given by one third the radiation energy density. We are therefore neglecting the effects of photon viscosity considered for example by Agol \& Krolik (1998). The radiation momentum equation (5) deserves special comment. We have assumed a simple diffusion form, and have in particular neglected time derivative and velocity-dependent terms that, e.g. Stone et al. (1992) and Gammie (1998) have retained. The full equation is

$$
\frac{1}{c^{2}}\left(\frac{\partial \mathbf{F}}{\partial t}+\mathbf{v} \cdot \nabla \mathbf{F}+\mathbf{F} \nabla \cdot \mathbf{v}\right)=-\frac{1}{3} \nabla E-\frac{\kappa_{\mathrm{es}} \rho}{c} \mathbf{F} .
$$

We are interested in perturbations with wavelengths $\lambda$ smaller than the vertical disk scale height $H$, and angular frequencies $\omega$ or growth rates which are of order the local angular velocity of the disk $\Omega$ (MRI, Balbus \& Hawley 1991) or possibly a factor $(2 \pi H / \lambda)^{1 / 2}$ larger (photon bubbles, Gammie 1998). All the terms on the left hand side of equation (7) will be negligible provided

$$
\frac{\omega}{\Omega} \ll \tau_{H}\left(\frac{c}{c_{\mathrm{s}}}\right) \text { and } \frac{v}{c} \ll \frac{\tau_{H}}{2 \pi}\left(\frac{\lambda}{H}\right),
$$

where $\tau_{H} \equiv \kappa_{\text {es }} \rho H$ is the scattering optical depth across a scale height, and $c_{s}$ is the total (gas plus radiation pressure) sound speed.

Well outside the innermost stable circular orbit, the scattering depth in the inner, radiation pressure dominated zone of a standard $\alpha$-viscosity disk (Shakura \& Sunyaev 1973) 1 is given by

$$
\tau_{H} \sim 1 \times \alpha^{-1} \eta\left(\frac{L}{\mathrm{~L}_{\mathrm{Edd}}}\right)^{-1}\left(\frac{R}{r_{\mathrm{g}}}\right)^{3 / 2} .
$$

Here $\eta \sim 0.1$ is the radiative efficiency, $L / \mathrm{L}_{\mathrm{Edd}}$ is the luminosity scaled with Eddington, and $r_{\mathrm{g}}$ is the gravitational radius of the central object. The scattering depth is therefore always significantly greater than unity except perhaps in the innermost parts of disks accreting near the Eddington limit. Radiative equilibrium, hydrostatic equilibrium, and the

\footnotetext{
${ }^{1}$ It is almost certainly the turbulence generated by the MRI which is responsible for "viscous" stresses in accretion disks, and therefore a value of $\alpha$. We are nevertheless using $\alpha$ to give us some handle on the ambient conditions in a fictitious, laminar accretion disk prior to the development of any instability. The reader should therefore view these scalings with appropriate caution.
} 
assumption that the viscous stress is $\alpha$ times the total pressure leads to a ratio of the speed of light to the sound speed $c_{\mathrm{s}} \simeq(4 E / 9 \rho)^{1 / 2}$ given by

$$
\frac{c}{c_{\mathrm{s}}} \sim \alpha \tau_{H}
$$

Hence conditions (8) are almost always satisfied in the interior of accretion disks, provided we do not consider wavelengths that are extremely small. The only exception is for near-Eddington disks in the innermost few gravitational radii, where our Newtonian treatment of the magnetohydrodynamics breaks down anyway. We therefore choose to neglect the left hand side of equation (77), which therefore reduces to the diffusion equation (5). The fact that Gammie (1998) found flux limited electromagnetic waves as solutions of his dispersion relation is a reflection of the fact that he retained these terms. In most cases, these terms have negligible effect on the photon bubble modes, which are at much lower frequency. N Note that equation (5) allows us to write the radiation pressure very simply in terms of a gradient in the radiation energy density $E$, so the momentum equation (2) becomes

$$
\rho\left(\frac{\partial \mathbf{v}}{\partial t}+\mathbf{v} \cdot \nabla \mathbf{v}\right)=-\nabla\left(p+\frac{1}{3} E\right)-\rho \nabla \Phi+\frac{1}{4 \pi}(\nabla \times \mathbf{B}) \times \mathbf{B} .
$$

Eliminating $\mathbf{F}$ using equation (5), we may write the radiation energy equation (4) as

$$
\frac{\partial E}{\partial t}+\mathbf{v} \cdot \nabla E+\frac{4}{3} E \nabla \cdot \mathbf{v}=\nabla \cdot\left(\frac{c}{3 \kappa_{\mathrm{es}} \rho} \nabla E\right) .
$$

In contrast to the radiation momentum equation (7), the left hand side of this equation can be neglected only if

$$
\frac{\omega}{\Omega} \ll \frac{4 \pi^{2}}{3 \tau_{H}}\left(\frac{c}{c_{\mathrm{s}}}\right) \sim \frac{4 \pi^{2}}{3} \alpha\left(\frac{H}{\lambda}\right)^{2} \text { and } \frac{v}{c} \ll \frac{2 \pi}{3 \tau_{H}}\left(\frac{H}{\lambda}\right) .
$$

Because $\alpha$ may be quite small and $\tau_{H}$ is not very small, these conditions may not be satisfied, so we must retain the left hand side of the radiation energy equation (4). In fact, it is neglecting the right hand side of this equation (photon diffusion) which returns us to the standard ideal MHD equations with gas and radiation pressure. When the conditions (13) start to become satisfied, photon diffusion starts to modify standard MHD. It is this effect which gives rise to photon bubbles, and which can modify the MRI. We therefore choose to retain the left hand side of equation (4), although the right hand side can be dominant.

\footnotetext{
${ }^{2}$ Indeed, we have numerically recomputed all the results in this paper with the terms on the left hand side of equation (7) included and found no significant differences for realistic physical parameters.
} 


\subsection{Equilibrium}

We consider a narrow annulus of the disk which, in equilibrium, is stationary and axisymmetric with a purely azimuthal flow velocity $\mathbf{v}=R \Omega \hat{\phi}$. (We assume cylindrical polar coordinates $\{R, \phi, z\}$ throughout this paper.) Following the usual analysis of the MRI as well as previous analyses of photon bubbles, we assume the existence of a locally uniform magnetic field. Because we are considering a differentially rotating system, we assume this field has no radial component, which would otherwise be sheared into a time-dependent azimuthal field. Hence $\mathbf{B}=B_{\phi} \hat{\boldsymbol{\phi}}+B_{z} \hat{\mathbf{z}}$. With the exception of the external gravitational potential $\Phi$, we assume that all radial gradients are much smaller than vertical gradients of the same quantities, i.e. that the disk is geometrically thin. The radiative diffusion equation (5) then implies that the equilibrium radiative flux is mainly in the vertical direction, and thus the radial component may be neglected. We will allow for the possibility of radial fluid gradients creating small departures from Keplerian flow by retaining a general form for the angular velocity profile $\Omega(R)$ in our dispersion relation.

With these assumptions, the only nontrivial equilibrium equations are those expressing vertical hydrostatic balance,

$$
-\frac{1}{\rho} \frac{\partial p}{\partial z}+\frac{\kappa_{\mathrm{es}}}{c} F_{z}-g=0
$$

radiative equilibrium,

$$
\frac{\partial F_{z}}{\partial z}=0
$$

and radiation diffusion,

$$
F_{z}=-\frac{c}{3 \kappa_{\mathrm{es}} \rho} \frac{\partial E}{\partial z} .
$$

Here $g \equiv \partial \Phi / \partial z$ is the magnitude of the local vertical gravitational acceleration produced by the tidal field of the central mass.

This equilibrium is characterized by a number of parameters which are important in describing wave modes. First are the gas and radiation sound speeds, defined as

$$
c_{\mathrm{g}}=\left(\frac{\gamma p}{\rho}\right)^{1 / 2} \text { and } c_{\mathrm{r}}=\left(\frac{4 E}{9 \rho}\right)^{1 / 2},
$$

respectively. Then there are the Alfvén speeds associated with each component of the magnetic field,

$$
v_{\mathrm{A} \phi}=\frac{B_{\phi}}{(4 \pi \rho)^{1 / 2}} \quad \text { and } \quad v_{\mathrm{A} z}=\frac{B_{z}}{(4 \pi \rho)^{1 / 2}}
$$


Internal hydrodynamic modes are driven by equilibrium gradients with associated oscillation frequencies. The square of the epicyclic frequency is given by

$$
\kappa^{2}=\frac{1}{R^{3}} \frac{d}{d R}\left(R^{4} \Omega^{2}\right)
$$

It is convenient to define the square of the Brunt-Väisälä frequency separately in the gas and radiation as

$$
N_{\mathrm{g}}^{2}=g\left[\frac{1}{\rho c_{\mathrm{g}}^{2}}\left(\frac{\partial p}{\partial z}\right)-\frac{\partial \ln \rho}{\partial z}\right]
$$

and

$$
N_{\mathrm{r}}^{2}=g\left[\frac{1}{3 \rho c_{\mathrm{r}}^{2}}\left(\frac{\partial E}{\partial z}\right)-\frac{\partial \ln \rho}{\partial z}\right],
$$

respectively. Then the square of the total Brunt-Väisälä frequency is

$$
N^{2}=g\left[\frac{1}{\rho\left(c_{\mathrm{g}}^{2}+c_{\mathrm{r}}^{2}\right)} \frac{\partial}{\partial z}\left(p+\frac{1}{3} E\right)-\frac{\partial \ln \rho}{\partial z}\right]=\frac{c_{\mathrm{g}}^{2} N_{\mathrm{g}}^{2}+c_{\mathrm{r}}^{2} N_{\mathrm{r}}^{2}}{c_{\mathrm{g}}^{2}+c_{\mathrm{r}}^{2}} .
$$

Finally, there is a characteristic length scale associated with radiative diffusion, given by how far photons diffuse on an orbital time. This can be used to define a diffusion wavenumber as

$$
k_{\mathrm{diff}} \equiv\left(\frac{3 \kappa \kappa_{\mathrm{es}} \rho}{c}\right)^{1 / 2}
$$

\subsection{Perturbation Equations}

Linearizing our equations of motion about the equilibrium, and assuming a WKB space-time dependence $\propto \exp \left[i\left(\int k_{R} d R+\int k_{z} d z-\omega t\right)\right]$, we arrive at the following perturbation equations:

$$
\begin{gathered}
-i \omega \delta \rho+i \rho \mathbf{k} \cdot \delta \mathbf{v}+\delta v_{z} \frac{\partial \rho}{\partial z}=0 \\
-i \omega \rho \delta v_{R}-2 \Omega \rho \delta v_{\phi}=-i k_{R}\left(\delta p+\frac{1}{3} \delta E\right)-i \frac{k_{R}}{4 \pi}\left[B_{\phi} \delta B_{\phi}+B_{z} \delta B_{z}\right]+i \frac{k_{z}}{4 \pi} B_{z} \delta B_{R} \\
-i \omega \rho \delta v_{\phi}+\frac{\kappa^{2}}{2 \Omega} \rho \delta v_{R}=i \frac{k_{z}}{4 \pi} B_{z} \delta B_{\phi} \\
-i \omega \rho \delta v_{z}=-i k_{z}\left(\delta p+\frac{1}{3} \delta E\right)-g \delta \rho-i \frac{k_{z}}{4 \pi} B_{\phi} \delta B_{\phi} \\
-i \omega \delta p+\delta v_{z} \frac{\partial p}{\partial z}=-i \gamma p \mathbf{k} \cdot \delta \mathbf{v} \\
-i \omega \delta E+\delta v_{z} \frac{\partial E}{\partial z}+i \frac{4}{3} E \mathbf{k} \cdot \delta \mathbf{v}=-i \mathbf{k} \cdot \delta \mathbf{F}
\end{gathered}
$$




$$
\begin{gathered}
\delta F_{R}=-\frac{i c}{3 \kappa_{e s} \rho} k_{R} \delta E \\
\delta F_{\phi}=0 \\
\delta F_{z}=-F_{z} \frac{\delta \rho}{\rho}-\frac{i c}{3 \kappa_{e s} \rho} k_{z} \delta E \\
-i \omega \delta B_{R}=i k_{z} B_{z} \delta v_{R}, \\
-i \omega \delta B_{\phi}-R \frac{d \Omega}{d R} \delta B_{R}=-i B_{\phi} \mathbf{k} \cdot \delta \mathbf{v}+i k_{z} B_{z} \delta v_{\phi},
\end{gathered}
$$

and

$$
-i \omega \delta B_{z}=-i k_{R} B_{z} \delta v_{R} .
$$

The radial and vertical induction equations together guarantee that the divergence of the magnetic field perturbation vanish. Hence one or the other can be replaced with

$$
i k_{R} \delta B_{R}+i k_{z} \delta B_{z}=0
$$

Equations (24) and (28)-(32) can be solved to yield a simple expression for the perturbed total pressure, viz.

$$
\delta p+\frac{1}{3} \delta E=\left(c_{\mathrm{g}}^{2}+\frac{\omega}{\omega_{\text {diff }}} c_{\mathrm{r}}^{2}\right) \delta \rho-\frac{\rho}{g}\left(c_{\mathrm{g}}^{2} N_{\mathrm{g}}^{2}+\frac{\omega}{\omega_{\text {diff }}} c_{\mathrm{r}}^{2} N_{\mathrm{r}}^{2}\right) \frac{i \delta v_{z}}{\omega}-\frac{k_{z} F_{z}}{3 \omega_{\text {diff }}} \frac{\delta \rho}{\rho},
$$

where

$$
\omega_{\text {diff }} \equiv \omega+i \frac{k^{2}}{k_{\text {diff }}^{2}} \kappa
$$

Each term on the right hand side of equation (37) has a simple interpretation. The first is the acoustic pressure perturbation in response to rarefaction or compression. The second is the buoyancy force (note that $i \delta v_{z} / \omega$ is simply the vertical Lagrangian displacement of a fluid element). The last term is the radiation pressure perturbation arising from the enhanced (diminished) optical depth in compression (rarefaction) regions. The middle term can give rise to convection, while the last term is responsible for photon bubble modes.

The twelve equations (24)-(34) and (36) relate twelve perturbation variables. Eight of these equations have an explicit $\omega$ dependence, and so we therefore expect an eighth order dispersion relation. However, as we show in Appendix A, one of these modes is in fact a zero frequency solution which merely represents a perturbation to the equilibrium. The interesting physics therefore lies in a seventh order dispersion relation. In section 4 , we will present this full dispersion relation, but we will first discuss in section 3 the simpler case of the MRI in a non-stratified equilibrium. 


\section{The Magnetorotational Instability in the Absence of Stratification}

In the fictitious laminar flow we are considering, it is presumably the MRI which generates the turbulence whose dissipation, combined with cooling, sets up the thermal profile which then might drive photon bubble and convective modes. We therefore begin by considering the MRI in isolation, but in a flow which is still hot enough that radiation pressure dominates gas pressure. In the absence of equilibrium stratification, the perturbation equations can be solved to yield the following dispersion relation:

$$
D_{\mathrm{ms}} D_{\mathrm{BH}}+k_{z}^{2} v_{\mathrm{A} z}^{2} v_{\mathrm{A} \phi}^{2}\left(k_{z}^{2} R \frac{d \Omega^{2}}{d R}-k^{2} \tilde{\omega}^{2}\right)-\frac{k_{R}^{2}}{k_{z}^{2}} \tilde{\omega}^{2} \omega^{4}=0 .
$$

Here

$$
\begin{gathered}
\tilde{\omega}^{2} \equiv \omega^{2}-k_{z}^{2} v_{\mathrm{A} z}^{2}, \\
D_{\mathrm{ms}} \equiv \omega^{2}-k_{z}^{2}\left(c_{\mathrm{g}}^{2}+\frac{\omega}{\omega_{\mathrm{diff}}} c_{\mathrm{r}}^{2}+v_{\mathrm{A} \phi}^{2}\right)
\end{gathered}
$$

are magnetosonic dispersion terms, and

$$
D_{\mathrm{BH}} \equiv \frac{k^{2}}{k_{z}^{2}} \tilde{\omega}^{4}-\kappa^{2} \tilde{\omega}^{2}-4 \Omega^{2} k_{z}^{2} v_{\mathrm{A} z}^{2}
$$

is the MRI dispersion relation (if set to zero) in a non-stratified, incompressible medium (Balbus \& Hawley 1991).

The dispersion relation (39) describes how the MRI is coupled to magnetosonic modes when allowance is made for compressibility and radiation. Without radiation, it reduces to equation (64) of Blaes \& Balbus (1994) for $k_{R}=0$, which is all they considered. The conclusion of those authors remains valid here: provided the equilibrium azimuthal magnetic field is sufficiently subthermal, with respect to either the gas or radiation energy densities, then the MRI remains essentially unchanged. Mathematically, this is because the Alfvén speed is then much smaller than the relevant sound speed. For the MRI, $|\omega| \sim \Omega \sim k v_{\mathrm{A} z}$, and the dispersion relation reduces to

$$
\left(c_{\mathrm{g}}^{2}+\frac{\omega}{\omega_{\text {diff }}} c_{\mathrm{r}}^{2}\right) D_{\mathrm{BH}}=0,
$$

so that the MRI mode frequencies remain unchanged from their incompressible form. Physically, in subthermal magnetic fields, the relevant wave speed for the MRI (the Alfvén speed) is much slower than the sound speed, so the associated fluid motions are very nearly incompressible. 
The question of how subthermal the azimuthal field must be in order not to affect the MRI is determined by radiative diffusion. From the form of the dispersion relation (39), it is clear that the MRI will be unaffected provided

$$
v_{\mathrm{A} \phi}^{2} \ll c_{\mathrm{g}}^{2}+c_{\mathrm{r}}^{2}\left(1+\frac{\Omega^{4}}{v_{\mathrm{A} z}^{4} k_{\mathrm{diff}}^{4}}\right)^{-1 / 2} .
$$

A sufficient condition for this to be true is that $v_{\mathrm{A} \phi} \ll c_{\mathrm{g}}$. However, in a radiation pressure dominated region of an accretion disk, it is reasonable to consider azimuthal fields for which $c_{\mathrm{g}}<v_{\mathrm{A} \phi}<c_{\mathrm{r}}$. In this case the MRI can be modified if the characteristic wavenumber for instability $\Omega / v_{\mathrm{A} z}$ exceeds the diffusion wavenumber $k_{\text {diff }}$, i.e. photons have time to diffuse across a wavelength in an orbital time. This reduces the effective sound speed, making the fluid more compressible. The criterion that the MRI be unaffected is more stringent: $v_{\mathrm{A} \phi} \ll c_{\mathrm{r}}\left(k_{\mathrm{diff}} v_{\mathrm{A} z} / \Omega\right)$.

Compressibility acts to reduce the growth rate of the MRI in the presence of azimuthal fields, but it does not remove the instability (Blaes \& Balbus 1994). This is illustrated in Fig. 1, which depicts the maximum growth rate as a function of azimuthal field strength and diffusion wavenumber. We have restricted consideration to vertical $\left(k_{R}=0\right)$ modes, as these are expected to have the fastest growth rates, and we consider an equilibrium with negligible gas pressure $\left(c_{\mathrm{g}}=0\right)$. At high values of $k_{\operatorname{diff}} v_{\mathrm{A} z} / \Omega$, photon diffusion is negligible, and we recover the MRI behavior for an ideal compressible fluid. Subthermal azimuthal fields then produce no effect on the growth rate of the instability, but when the field starts to become thermal in strength, there is a modest reduction in the growth rate. For $k_{\mathrm{diff}} v_{\mathrm{A} z} / \Omega \lesssim 1$, photon diffusion is important. In this regime, azimuthal fields can reduce the growth rate quite substantially if $v_{\mathrm{A} \phi} \gtrsim c_{\mathrm{r}}\left(k_{\mathrm{diff}} v_{\mathrm{A} z} / \Omega\right)$. For the parameters chosen in the figure, this corresponds to $v_{\mathrm{A} \phi} / v_{\mathrm{A} z} \gtrsim 1$ for $k_{\mathrm{diff}} v_{\mathrm{A} z} / \Omega=0.1$. Higher values of $k_{\mathrm{diff}} v_{\mathrm{A} z} / \Omega$ (less diffusion) require higher azimuthal field strengths to affect the instability.

We can estimate how small the MRI growth rate becomes when diffusion is important by setting $k_{R}=c_{\mathrm{r}}=0$ in equation (39) and then solving it in the limit $c_{\mathrm{g}} \rightarrow 0$. The MRI then satisfies

$$
\omega^{2} \simeq \frac{k^{2} c_{\mathrm{g}}^{2} v_{\mathrm{A} z}^{2}\left(R \frac{d \Omega^{2}}{d R}+k^{2} v_{\mathrm{A} z}^{2}\right)}{v_{\mathrm{A} z}^{2}\left(R \frac{d \Omega^{2}}{d R}+k^{2} v_{\mathrm{A} z}^{2}\right)+v_{\mathrm{A} \phi}^{2}\left(\kappa^{2}+k^{2} v_{\mathrm{A} z}^{2}\right)}
$$

\footnotetext{
${ }^{3}$ We do not have a rigorous proof that the actual stability criterion remains unchanged in our case. However, we suspect that this is true based on our numerical calculations illustrated in Fig. 1. In addition, the dispersion relation (39) admits a zero frequency root for $\omega$ when $k^{2} v_{\mathrm{A} z}^{2}=-R\left(d \Omega^{2} / d R\right.$ ) (see section 4 ), which is the condition for the marginally stable wavenumber of the MRI in incompressible MHD in the absence of stratification.
} 
For $v_{\mathrm{A} \phi} \gg v_{\mathrm{A} z}$, the unstable growth rate is therefore reduced to $\sim \Omega\left(c_{\mathrm{g}} / v_{\mathrm{A} \phi}\right)$.

Note that regardless of photon diffusion, the MRI is virtually unaffected by compressibility provided the field is sufficiently vertical, i.e. $v_{\mathrm{A} \phi} \ll v_{\mathrm{A} z}$ (e.g. the

$v_{\mathrm{A} \phi} / v_{\mathrm{A} z}=0.1$ curve shown in Fig. 1). This is because a flow with a purely vertical equilibrium field will have vertical $\left(k_{R}=0\right)$ MRI modes identical to the incompressible case, as the dispersion relation (39) permits $D_{\mathrm{BH}}=0$ as a solution.

To summarize, the MRI is essentially unaffected by photon diffusion, unless the azimuthal component of the field is comparable to or larger than the vertical component, and the vertical component of the field is weak enough that photon diffusion is important on the scale of an unstable wavelength $\sim v_{\mathrm{A} z} / \Omega$. While the instability still persists, even subthermal azimuthal field components can then reduce the growth rate to $\sim \Omega\left(c_{\mathrm{g}} / v_{\mathrm{A} \phi}\right)$ if condition (44) is violated.

\subsection{The Relation Between the MRI, Alfvén and Slow Waves}

In the next section we will examine stratified equilibria, which turn out to be subject to a number of unstable modes which can couple to each other in complicated ways. To help disentangle this complexity, it is helpful to briefly consider the relation between the MRI and classical MHD waves.

Normally, in the absence of radiative diffusion, the MRI can be viewed as a differential rotation driven destabilization of the slow magnetosonic mode in a weakly magnetized medium (Balbus \& Hawley 1998). The distinction between slow and Alfvén modes is delicate, however. In the limit where the magnetic field becomes very weak, the slow magnetosonic mode becomes incompressible and has a dispersion relation which is the same as that of Alfvén waves. It is in fact identical in this limit to the "pseudo-Alfvén" mode which exists in incompressible fluids. The prefix "pseudo" is used because, although the mode is degenerate with the Alfvén mode, the eigenfunction is still different. Alfvén waves have velocity perturbations which are perpendicular to the plane of the wave vector and equilibrium magnetic field. Slow, or pseudo-Alfvén, waves have velocity perturbations in the plane of the wave vector and equilibrium magnetic field. For example, if $B_{\phi}=0$ in our geometry, Alfvén waves would involve only $\delta v_{\phi}$, whereas slow waves would have nonzero $\delta v_{R}$ and $\delta v_{z}$.

When the Coriolis forces associated with rotation are included, radial and azimuthal motions are coupled together, so the distinction between slow and Alfvén modes is blurred further. It is therefore perhaps accidental that the stable modes to which the MRI converts 
are normally the slow modes at high wavenumber. Figure 2(a) illustrates this fact in the case of negligible radiative diffusion. However, when radiative diffusion is important, the MRI can convert to the Alfvén wave [Fig. 2(b)] or the slow wave [Fig. 2(c)].

This would only be of academic interest were it not for the fact that it is the slow mode which continuously transforms at low wavenumbers into the unstable photon bubble mode in static, stratified equilibria (Gammie 1998). The relationship between this instability and the MRI in linear theory therefore depends on whether the Alfvén or slow mode is more closely tied to the MRI. Also, as we will show in the next section, the Alfvén mode is itself unstable in stratified equilibria.

\section{The Dispersion Relation in the Presence of Vertical Stratification}

With considerable algebra, the general perturbation equations from section 2 can be used to derive the following seventh order dispersion relation:

$$
\begin{aligned}
& D_{\mathrm{ms}} D_{\mathrm{BH}}+k_{z}^{2} v_{\mathrm{A} z}^{2} v_{\mathrm{A} \phi}^{2}\left(k_{z}^{2} R \frac{d \Omega^{2}}{d R}-k^{2} \tilde{\omega}^{2}\right)-\frac{k_{R}^{2}}{k_{z}^{2}} \tilde{\omega}^{2} \omega^{4} \\
& +\left(D_{\mathrm{BH}}-\frac{k_{R}^{2} \omega^{2} \tilde{\omega}^{2}}{k_{z}^{2}}\right)\left[-\frac{k_{z} k_{R}^{2} F_{z}}{3 \omega_{\mathrm{diff}} \rho}+\left(g-i \frac{k_{z}^{2} F_{z}}{3 \omega_{\mathrm{diff}} \rho}\right) \frac{1}{\rho} \frac{\partial \rho}{\partial z}\right] \\
& +i 2 \Omega \omega k_{z} k_{R} v_{\mathrm{A} z} v_{\mathrm{A} \phi}\left[-\frac{k_{z} k^{2} F_{z}}{3 \omega_{\mathrm{diff}} \rho}-i \frac{k_{z}^{2} F_{z}}{3 \omega_{\mathrm{diff}} \rho} \frac{1}{\rho} \frac{\partial \rho}{\partial z}-2 i k_{z} g\right] \\
& +k_{R}^{2} \tilde{\omega}^{2}\left(c_{\mathrm{g}}^{2} N_{\mathrm{g}}^{2}+\frac{\omega}{\omega_{\mathrm{diff}}} c_{\mathrm{r}}^{2} N_{\mathrm{r}}^{2}\right)+\frac{k_{R}^{2} k_{z} \tilde{\omega}^{2} F_{z} \omega^{2}}{3 \omega_{\mathrm{diff}} \rho}-\frac{g}{\rho} \frac{\partial \rho}{\partial z} k_{R}^{2} v_{\mathrm{A} \phi}^{2} \omega^{2}=0 .
\end{aligned}
$$

The first three terms are identical to the non-stratified dispersion relation (39) discussed in the last section. All other terms arise from the stratification. Note that in order for the WKB approximation to be consistent, we must have $\left|k_{z}\right| \gg|\partial \ln \rho / \partial z|$, so some of the terms with explicit dependence on the vertical density gradient should probably be taken to be negligible. In a radiation pressure dominated accretion disk, the WKB approximation also demands that

$$
k \gg \frac{g}{c_{\mathrm{r}}^{2}} \simeq \frac{\kappa_{\mathrm{es}} F_{z}}{c c_{\mathrm{r}}^{2}}
$$

The dispersion relation (46) reduces to those of previous authors under various limits. First, in the incompressible limit where $\left(c_{\mathrm{g}}^{2}+\omega c_{\mathrm{r}}^{2} / \omega_{\text {diff }}\right) \rightarrow \infty$, we recover the MRI dispersion relation in the presence of vertical stratification (Balbus \& Hawley 1991), albeit slightly 
modified by radiative diffusion:

$$
\frac{k^{2}}{k_{z}^{2}} \tilde{\omega}^{4}-\left[\kappa^{2}+\frac{k_{R}^{2}}{k_{z}^{2}}\left(\frac{c_{\mathrm{g}}^{2} N_{\mathrm{g}}^{2}+\omega c_{\mathrm{r}}^{2} N_{\mathrm{r}}^{2} / \omega_{\text {diff }}}{c_{\mathrm{g}}^{2}+\omega c_{\mathrm{r}}^{2} / \omega_{\text {diff }}}\right)\right] \tilde{\omega}^{2}-4 \Omega^{2} k_{z}^{2} v_{\mathrm{A} z}^{2}=0 .
$$

In addition, if we first assume that gas pressure is negligible $\left(c_{\mathrm{g}}^{2} \rightarrow 0\right)$, adopt the ansatz that $\omega \sim k^{1 / 2}$ and then take the short wavelength limit $k \rightarrow \infty$, we obtain two roots given by

$$
\omega^{2}=-i g\left(\frac{k_{z} k_{R}^{2}}{k^{2}}\right)\left(\frac{B_{z}}{B}\right)^{2} .
$$

This is identical to the asymptotic photon bubble instability dispersion relation obtained by Gammie (1998, eq. 41), adapted to our geometry. In spite of the presence of rotation, the photon bubble instability survives unscathed in this limit.

Finally, if we set the equilibrium magnetic field equal to zero, the equilibrium density to be constant, and the gas pressure to be negligible, then after cancelling two resulting zero modes, the dispersion relation reduces to fifth order:

$$
3 \omega^{5}+i \frac{k^{2} c}{\kappa_{\mathrm{es}} \rho} \omega^{4}-\left(3 \kappa^{2}+\frac{4 k^{2} E}{3 \rho}\right) \omega^{3}-i \frac{k^{2} c \kappa^{2}}{\kappa_{\mathrm{es}} \rho} \omega^{2}+\left(\frac{4 k_{z}^{2} \kappa^{2} E}{3 \rho}-3 k_{R}^{2} g^{2}\right) \omega+\frac{k_{z} k_{R}^{2} g c \kappa^{2}}{\kappa_{\mathrm{es}} \rho}=0 .
$$

This equation resembles the dispersion relation presented by Pietrini \& Krolik (2000) when the dissipation terms that they included are neglected. Their dispersion relation still has several additional terms compared to equation (50), however. Each of these terms are small and arise from the left hand side of the full radiation momentum equation (7), which we neglected.

Despite the complexity of the general dispersion relation, the MRI turns out to be a robust instability. An analytic indication that this is so can be found by just setting $\omega=0$ in equation (46), which leads to the following equation:

$$
k_{z}^{2} v_{\mathrm{A} z}^{2}\left(k^{2} v_{\mathrm{A} z}^{2}+R \frac{d \Omega^{2}}{d R}\right)\left[i \frac{k_{z} k_{R}^{2} F_{z} k_{\text {diff }}^{2}}{3 \rho k^{2} \kappa}+\left(g-\frac{k_{z}^{2} F_{z} k_{\text {diff }}^{2}}{3 \rho k^{2} \kappa}\right) \frac{1}{\rho} \frac{\partial \rho}{\partial z}-k_{z}^{2} c_{\mathrm{g}}^{2}\right]-k_{R}^{2} k_{z}^{2} v_{\mathrm{A} z}^{2} c_{\mathrm{g}}^{2} N_{\mathrm{g}}^{2}=0 .
$$

If the gas Brunt-Väisälä frequency $N_{\mathrm{g}}=0$, this equation shows that there will exist a zero frequency root if $k<k_{\text {crit }} \equiv\left[-R\left(d \Omega^{2} / d R\right)\right]^{1 / 2} / v_{\mathrm{A} z}$, the usual critical wavenumber for the onset of the MRI. Even if $N_{\mathrm{g}} \neq 0$, there will be a zero frequency root for $k_{R}=0$ and $k^{2} v_{\mathrm{A} z}^{2}=-R\left(d \Omega^{2} / d R\right)$. While not a rigorous proof, these facts suggest that the critical wavenumber for the onset of the MRI is unchanged by the effects of radiation. Hence the instability criterion for the MRI is still that the angular velocity decreases outward in magnitude. These expectations are born out by our numerical calculations. Radiative diffusion does not affect the stability criterion, although it can dramatically affect the growth rates, as discussed in section 3 . 


\subsection{Photon Bubble Modes and Other MHD Wave Instabilities}

In the high wavenumber limit $k \rightarrow \infty$, the dispersion relation can be factored to give

$$
\left(\omega+i \frac{k^{2} c}{3 \kappa_{\mathrm{es}} \rho}\right)\left(\omega^{2}-k_{z}^{2} v_{\mathrm{A} z}^{2}\right)\left[\omega^{4}-\omega^{2} k^{2}\left(c_{\mathrm{g}}^{2}+v_{\mathrm{A} z}^{2}+v_{\mathrm{A} \phi}^{2}\right)+k_{z}^{2} v_{\mathrm{A} z}^{2} k^{2} c_{\mathrm{g}}^{2}\right]=0 .
$$

The first factor is a strongly damped radiation diffusion mode, the second describes Alfvén waves, and the third corresponds to the fast and slow magnetosonic waves. Rotation is irrelevant at these high wavenumbers. We have lost the radiation effects on the fast and slow modes because in this limit the wavelength is much less than the diffusion length scale.

It is worthwhile examining the behavior of the MHD wave modes to higher order in $k^{-1}$, because they all turn out to be unstable for sufficiently high vertical photon fluxes. If, as we have been generally assuming, the radiation and magnetic energy densities in the plasma are much larger than the gas thermal energy density, then the fast and slow modes have frequencies given by

$$
\omega \simeq \pm k\left(v_{\mathrm{A} z}^{2}+v_{\mathrm{A} \phi}^{2}\right)^{1 / 2}-i \frac{3 \kappa_{\mathrm{es}} \rho}{2 c\left(v_{\mathrm{A} z}^{2}+v_{\mathrm{A} \phi}^{2}\right)}\left[c_{\mathrm{r}}^{2}\left(\frac{k_{R}^{2}}{k^{2}} v_{\mathrm{A} z}^{2}+v_{\mathrm{A} \phi}^{2}\right) \mp \frac{k_{z} k_{R}^{2} F_{z} v_{\mathrm{A} z}^{2}}{3 k^{3} \rho\left(v_{\mathrm{A} z}^{2}+v_{\mathrm{A} \phi}^{2}\right)^{1 / 2}}\right]
$$

and

$$
\omega \simeq \pm k_{z} c_{\mathrm{g}}\left(\frac{B_{z}}{B}\right) \mp i \frac{\kappa_{\mathrm{es}} k_{R}^{2} F_{z}}{2 c c_{\mathrm{g}} k^{2}}\left(\frac{B_{z}}{B}\right),
$$

respectively. The lowest order correction to the Alfvén mode is of order $k^{-1}$, and this correction is real, implying that any instability or damping is at still higher order. As we shall see, instabilities in the Alfvén waves peak at finite wavenumbers.

The first imaginary term in equation (53) represents damping of the fast modes by radiative diffusion. This term agrees with the optically thin damping rate derived by Agol \& Krolik (1998), although the waves need not be optically thin for this rate to apply. The last term in equation (53) shows that a sufficiently large vertical radiative flux can overcome the damping and destabilize the fast waves. The instability criterion can be obtained by comparing the last two terms of equation (53):

$$
F_{z}>3 \rho c_{\mathrm{r}}^{2}\left(1+\frac{k^{2} v_{\mathrm{A} \phi}^{2}}{k_{R}^{2} v_{\mathrm{A} z}^{2}}\right) \frac{k}{k_{z}}\left(v_{\mathrm{A} z}^{2}+v_{\mathrm{A} \phi}^{2}\right)^{1 / 2} .
$$

Crudely, if the speed at which photons are diffusing upward in the equilibrium exceeds the Alfvén speed, then fast modes propagating upward at some nonzero angle to the vertical will be unstable. For large photon fluxes, the growth rate of this instability is

$$
\operatorname{Im}(\omega) \simeq \frac{g}{2 v_{A}}\left(\frac{k_{z} k_{R}^{2}}{k^{3}}\right)\left(\frac{B_{z}}{B}\right)^{2} .
$$


The instability criterion is most easily satisfied, and the largest growth rates are achieved, for vertical magnetic fields. Azimuthal fields tend to be stabilizing.

Equation (54) reveals that slow modes propagating downward at some nonzero angle to the vertical are also unstable at high photon fluxes. These modes have the fastest growth rates, and at lower values of $k$ become the photon bubble modes described by Gammie's (1998) dispersion relation (49). Adopting Gammie's (1998) photon bubble ansatz of $\omega \sim k^{1 / 2}$ and retaining only the leading order terms as $k \rightarrow \infty$ in the general dispersion relation, we find

$$
\omega^{2}=\left(k_{z}^{2} c_{\mathrm{g}}^{2}-i \frac{k_{z} k_{R}^{2} \kappa_{\mathrm{es}} F_{z}}{c k^{2}}-i \omega \frac{3 \kappa_{\mathrm{es}} \rho c_{\mathrm{r}}^{2} k_{z}^{2}}{c k^{2}}\right)\left(\frac{B_{z}}{B}\right)^{2}
$$

The middle term gives the photon bubble dispersion relation (49) when use is made of the hydrostatic equilibrium condition (14) in the radiation dominated limit. The other terms compete with the photon bubble term at low and high wavenumbers, leading us to expect that the photon bubble dispersion relation (49) will only hold in the range of wavenumbers given by

$$
\frac{9 \rho^{2} c_{\mathrm{r}}^{2} \kappa_{\mathrm{es}}^{2}}{c^{2} g} \lesssim k \lesssim \frac{g}{c_{\mathrm{g}}^{2}}
$$

A condition equivalent to this was also noted by Gammie (1998). When the last inequality breaks down at high wavenumbers, the slow mode dispersion relation is better described by equation (54).

Figure 3 presents a numerical solution to the full dispersion relation (46) which illustrates these high wavenumber instabilities and compares their growth rates to the analytic expressions above. In addition to the fast and slow modes, the Alfvén modes also appear to go unstable for large photon fluxes. In contrast to the fast and slow modes, the Alfvén wave growth rates tend to zero as $k \rightarrow \infty$. Rotation directly affects the unstable growth rates of the Alfvén modes, and also affects the fast and slow modes at low wavenumbers. We describe this, and the coupling to the MRI, in the next subsections.

\subsection{Zero Azimuthal Field Case}

We now return to low wavenumbers where rotation is important. We first consider the case where the equilibrium field is vertical $\left(B_{\phi}=0\right)$. The general dispersion relation (46) then reduces to

$$
D_{\mathrm{ms}} D_{\mathrm{BH}}-\frac{k_{R}^{2}}{k_{z}^{2}} \tilde{\omega}^{2} \omega^{4}+\left(D_{\mathrm{BH}}-\frac{k_{R}^{2} \omega^{2} \tilde{\omega}^{2}}{k_{z}^{2}}\right)\left[-\frac{k_{z} k_{R}^{2} F_{z}}{3 \omega_{\mathrm{diff}} \rho}+\left(g-i \frac{k_{z}^{2} F_{z}}{3 \omega_{\mathrm{diff}} \rho}\right) \frac{1}{\rho} \frac{\partial \rho}{\partial z}\right]
$$




$$
+k_{R}^{2} \tilde{\omega}^{2}\left(c_{\mathrm{g}}^{2} N_{\mathrm{g}}^{2}+\frac{\omega}{\omega_{\mathrm{diff}}} c_{\mathrm{r}}^{2} N_{\mathrm{r}}^{2}\right)+\frac{k_{R}^{2} k_{z} \tilde{\omega}^{2} F_{z} \omega^{2}}{3 \omega_{\text {diff }} \rho}=0 .
$$

Note that $D_{\mathrm{BH}}=0$ is a solution of this equation when $k_{R}=0$, so we immediately conclude that in the absence of an azimuthal field, the vertical MRI modes are completely unaffected by compressibility, stratification, and radiation. This is not surprising, as the vertical MRI modes involve horizontal motions only, and therefore do not involve any excess forces from the vertical radiative flux.

Equation (59) is complicated, with many parameters. For numerical computations, we restrict consideration to cases where the gas pressure is negligible, so that $c_{\mathrm{g}}$ and $N_{\mathrm{g}}$ are both zero. In addition, we neglect convection and couplings to gravity modes by considering only isentropic equilibria, so that $N_{\mathrm{r}}=0$. Under these assumptions, we may use equation (14) to write the acceleration due to gravity and the equilibrium vertical density gradient in terms of the photon flux, viz.

$$
g=\frac{k_{\mathrm{diff}}^{2}}{3 \kappa \rho} F_{z} \text { and } \frac{1}{\rho} \frac{\partial \rho}{\partial z}=-\frac{k_{\mathrm{diff}}^{2}}{3 \kappa \rho c_{\mathrm{r}}^{2}} F_{z} .
$$

Hence the only new parameter is $F_{z}$.

Figure 4 presents numerical calculations of the unstable mode growth rates in the diffusive regime at low wavenumber, for various values of photon flux. The introduction of stratification causes the slow (photon bubble) and Alfvén wave instabilities to appear and have increasing growth rate as $F_{z}$ gets larger. The fast wave instability appears only after a critical threshold in $F_{z}$ is reached [cf. Fig. 4(b)]. This is quantitatively consistent with our instability criterion (55), which predicts that the fast wave will be unstable for $F_{z} /\left(3 \rho v_{\mathrm{A} z}^{3}\right)>125$ for the parameters chosen in Fig. 4. Fig. 4(c) should be compared with Fig. 3 which has similar radiation parameters. Note that for the value of $k_{\text {diff }}$ chosen in Fig. 4, the MRI converts to Alfvén waves at $k_{\text {crit }}[\mathrm{cf}$. Fig. 2(b)], and indeed the unstable Alfvén waves show up for $k>k_{\text {crit }}$. The MRI itself appears to be largely unaffected by stratification, except that the maximum growth rate increases with increasing $F_{z}$. It is unclear what would happen at higher fluxes because we are already close to the WKB limit (47) at these high fluxes. Given that the vertical $\left(k_{R}=0\right)$ MRI modes are unaffected by flux, they are not always the fastest growing modes in the presence of nonzero flux. Modes with a slight nonzero $k_{R}$ in fact grow faster for the equilibrium parameters shown in Fig. $4(\mathrm{c})$. The fact that $F_{z}$ can affect the MRI growth rates for $k_{R} \neq 0$, albeit modestly, suggests that geometrically thick accretion flows with significant radial flux in the equilibrium could have a more marked effect on the MRI than the geometrically thin configurations considered in this paper.

The photon bubble mode is always the most rapidly growing of the three MHD wave 
instabilities that exist in stratified media. It appears to survive for $k<k_{\text {crit }}$, albeit at a smaller growth rate than expected from extrapolation of the high $k$ behavior. Rotation therefore acts to mildly suppress, and does not kill, this instability when $B_{\phi}=0$.

\subsection{Nonzero Azimuthal Field}

We finally turn to the full dispersion relation (46) with nonzero azimuthal field, but continuing to neglect gas pressure and vertical entropy gradients. Figure 5 presents results for the low wavenumber instability growth rates corresponding the the $B_{\phi}=0$ case shown in Fig. 4(b). As we stated above, the MRI is a robust instability which even in the presence of a nonzero azimuthal field and radiation, still exists for $k<k_{\text {crit }}$. However, as expected from our results in section 3 , the nonzero azimuthal field produces a marked reduction in the overall growth rate. The maximum MRI growth rates in Fig. 5 are comparable to the $k_{R}=0$ growth rates shown in Fig. 1 at the chosen diffusion wavenumber $k_{\operatorname{diff}} v_{\mathrm{A} z} / \Omega=0.1$. The vertical radiative flux makes little difference to the MRI compared to the nonstratified case, just as we found in the previous subsection.

The azimuthal field component has a noticeable effect on the other instabilities. First, the fast and Alfvén waves are quickly stabilized. This is consistent with our findings in section 4.1, where we noted that azimuthal fields would stabilize the fast waves. For the parameters chosen in Fig. 5, equation (55) predicts fast wave stability for $B_{\phi} \gtrsim 0.4 B_{z}$.

The photon bubble growth rate also decreases with increasing azimuthal field, in quantitative agreement with equation (49). At high values of $B_{\phi} / B_{z}$, this mode appears to split into a high wavenumber part (the true photon bubble), and a low wavenumber part [cf. Fig. 5(b)]. The latter dominates the MRI growth rate except at the lowest wavenumbers, at least for the parameters chosen in Fig. 5. At high wavenumbers, this mode evolves into the Alfvén mode.

\section{Astrophysical Relevance}

It turns out that diffusion is actually very important in standard $\alpha$-viscosity models of accretion disks around black holes and neutron stars (Shakura \& Sunyaev 1973). Neglecting relativistic correction factors, one can show that in the radiation pressure dominated inner zone,

$$
k_{\text {diff }} H \simeq 2 \alpha^{-1 / 2} \text { radians. }
$$


Surprisingly, this relation is independent of accretion rate, radius, and central object mass. Combining this with the WKB condition for the MRI, $k \sim \Omega / v_{\mathrm{A} z} \gg H^{-1}$, we find

$$
\frac{k_{\mathrm{diff}} v_{\mathrm{A} z}}{\Omega} \ll \alpha^{-1 / 2} .
$$

Depending on the assumed strength of the initial magnetic field, realistic accretion disk models $(\alpha \sim 0.01-0.1)$ will often be in the regime where photon diffusion can significantly slow the growth of the MRI in the presence of azimuthal field components (cf. Fig. 1). Our analysis shows that in this case the growth rate would be reduced to $\sim \Omega\left(c_{\mathrm{g}} / v_{\mathrm{A} \phi}\right)$. In the radiation pressure dominated inner zone, one can conceive of $v_{\mathrm{A} \phi}$ being somewhat less than $c_{\mathrm{r}}$. Neglecting relativistic correction factors once again,

$$
\frac{c_{\mathrm{g}}}{c_{\mathrm{r}}} \simeq 4 \times 10^{-3} \alpha^{-1 / 8} \eta\left(\frac{M}{\mathrm{M}_{\odot}}\right)^{-1 / 8}\left(\frac{L}{\mathrm{~L}_{\mathrm{Edd}}}\right)\left(\frac{R}{r_{\mathrm{g}}}\right)^{21 / 16}
$$

where $M$ is the mass of the central object. The MRI growth rate can therefore become quite sluggish if the azimuthal field energy density exceeds the thermal energy density in the gas by an amount comparable to the radiation/gas thermal energy density ratio. We stress however that even in this regime, the MRI still exists as a dynamical instability, and will still presumably grow eventually to nonlinear amplitude. It might be interesting to see if the resulting turbulence depends on how the initial magnetic field energy density compares to the gas and radiation thermal energy densities, but it is difficult to predict any results from linear theory alone.

Note that all these conclusions refer to a fictitious, unstable initial state which cannot exist in nature. The equations above, in particular that for the diffusion wavenumber (62), depend on $\alpha$, which is presumably a consequence of the MRI. It is therefore difficult to determine how the MRI will be affected when it is responsible for the ambient conditions in the disk in the first place.

This fact is even more important when we consider the stratified calculations that we presented in section 4. Multiple instabilities are simultaneously present in this case, and the modes with the fastest growth rates presumably dominate the nonlinear evolution. One would at first conclude that the photon bubble/slow mode at high wavenumbers therefore controls the dynamics. From equation (54), the growth rate is at most

$$
\operatorname{Im}(\omega) \simeq 10^{2} \Omega \alpha^{1 / 8} \eta^{-1}\left(\frac{L}{\mathrm{~L}_{\mathrm{Edd}}}\right)\left(\frac{M}{\mathrm{M}_{\odot}}\right)^{1 / 8}\left(\frac{R}{r_{\mathrm{g}}}\right)^{-21 / 16}
$$

substantially faster than the orbital frequency $\Omega$. 
However, it is important to recognize the different sources of free energy which are driving these instabilities. The MRI feeds off the differential rotation in the flow, transporting angular momentum outward which ultimately allows material to accrete and release gravitational potential energy. We therefore view this instability as being fundamental to the dynamics of the accretion disk. Dissipation of the gravitational energy released and radiative transport processes set up vertical gradients which drive the photon bubble and other MHD wave instabilities. Convection will also presumably occur if the resulting entropy gradient has the right sign (Pietrini \& Krolik 2000). It is the vertical gradients which provide the source of free energy for these extra instabilities, but these gradients are fundamentally determined by the action of the MRI.

We speculate that on the largest vertical scales, where diffusion is negligible on an orbital time, the MRI will always operate as usual, launching perhaps a turbulent cascade to smaller scales. How this energy is ultimately dissipated is still unclear. If there is sufficient vertical flux, it may not be as simple as radiative damping of compressive MHD waves (Agol \& Krolik 1998), as those waves are themselves unstable for some propagation directions. Given the high growth rates at short wavelengths from equation (64) above, it seems likely to us that photon bubbles will play an important role in thermal transport on small scales.

Concrete answers to the question of how radiative diffusion and radiative flux really affect the turbulent angular momentum and heat transport in the disk will require investigation by numerical simulations. One must be careful to choose the fictitious unstable initial state with care when pursuing such simulations, keeping in mind that accretion power, and therefore the MRI, is the driver for all this complex dynamics.

\section{Conclusions}

We may summarize our conclusions as follows. In the absence of an azimuthal field, the vertical $\left(k_{R}=0\right)$ MRI modes have the standard, incompressible behavior elucidated by Balbus \& Hawley (1991). If the azimuthal field component dominates the vertical field component and the Alfvén speed is larger than the effective sound speed [cf. eq. (44)], then the growth rate of the MRI is reduced to $\sim \Omega\left(c_{\mathrm{g}} / v_{\mathrm{A} \phi}\right)$, well below the usual rapid growth rate of order the orbital frequency $\Omega$. However, the instability still persists. Large vertical photon fluxes in the equilibrium do not significantly affect the most rapidly growing MRI modes.

At short wavelengths where rotation is unimportant, both the fast and slow MHD 
waves are destabilized by sufficiently high vertical photon flux in the equilibrium. These instabilities require that the waves propagate at some nonzero angle to the vertical, and affects upward propagating fast waves and downward propagating slow waves. The slow waves appear to always have the larger growth rates. At smaller wavenumbers these are well-described by Gammie's (1998) photon bubble dispersion relation (49), but at larger wavenumbers they asymptote to a maximum value given by equation (54). At small wavenumbers where rotation is important $\left(k<k_{\text {crit }}\right)$, the photon bubble growth rate is somewhat suppressed by rotation, at least at modest fluxes. Nonzero azimuthal fields stabilize the fast wave instability and reduce the photon bubble growth rate. In addition to the fast and slow wave instabilities, the vertical radiative flux also drives unstable modes at intermediate wavenumber which are related to Alfvén waves at high wavenumber.

The ambient conditions in the radiation pressure dominated inner region of standard alpha disk models are generally in a regime where radiative diffusion can slow the growth of the MRI. In addition, photon bubble modes are expected at short wavelengths with growth rates that can significantly exceed the orbital frequency. It is not clear how these instabilities will evolve into the nonlinear, turbulent regime, but we speculate that the MRI will dominate the largest scales and that photon bubbles (and perhaps unstable fast and Alfvén waves) may affect both the dissipation and thermal transport on smaller scales. A complete understanding of the turbulent angular momentum and heat transport will require numerical simulations.

Finally, we stress that we have concentrated here on geometrically thin accretion disk configurations where the photon flux is primarily in the vertical direction and the rotation curve is near-Keplerian. Geometrically thick or quasi-spherical, radiation pressure supported flows will have radiation pressure gradient components in the radial direction and may therefore exhibit more interesting dynamical coupling between the radiative flux and the MRI.

We acknowledge useful conversations with Eric Agol, Yuri Levin, and Jim Stone. This work was supported by NSF grants AST-9970827 and PHY-9907949, and NASA grant NAG5-7075.

\section{A. Appendix A: The Zero Frequency Mode of the General Dispersion Relation}

Despite the fact that the perturbation equations (24)-(34) and (36) have eight separate $\omega$-dependent terms, we only end up with a dispersion relation that is seventh order. The 
reason is that there is a zero frequency mode, which we discuss in some detail in this appendix.

Setting $\omega=0$ in the perturbation equations, the radial induction equation (33) immediately implies that $\delta v_{R}$ must vanish. The continuity equation (24) and azimuthal momentum equation (26) then imply that $\delta v_{z}$ and $\delta B_{\phi}$ must also vanish. The gas energy equation (28) is then trivially satisfied, which is the mathematical reason why a zero frequency mode is a possible solution of the perturbation equations.

Excluding the trivial azimuthal flux equation (31), the remaining seven equations may be written

$$
\begin{gathered}
-2 \Omega \rho \delta v_{\phi}+i k_{R}\left(\delta p+\frac{1}{3} \delta E\right)+i \frac{k_{R}}{4 \pi} B_{z} \delta B_{z}-i \frac{k_{z}}{4 \pi} B_{z} \delta B_{R}=0, \\
i k_{z}\left(\delta p+\frac{1}{3} \delta E\right)+g \delta \rho=0, \\
\mathbf{k} \cdot \delta \mathbf{F}=0, \\
\delta F_{R}+\frac{i c}{3 \kappa_{e s} \rho} k_{R} \delta E=0, \\
\delta F_{z}+F_{z} \frac{\delta \rho}{\rho}+\frac{i c}{3 \kappa_{e s} \rho} k_{z} \delta E=0, \\
-R \frac{d \Omega}{d R} \delta B_{R}-i k_{z} B_{z} \delta v_{\phi}=0,
\end{gathered}
$$

and

$$
k_{r} \delta B_{R}+k_{z} \delta B_{z}=0 .
$$

These seven equations have eight unknowns, so that any one of the nonzero perturbation amplitudes determine the other seven for this zero frequency mode.

It is perhaps surprising that this mode has such a complicated eigenfunction, and it is therefore worthwhile understanding its physical meaning. Equation (A6) is particularly interesting. This arose from the azimuthal component of the induction equation which describes, for example, how a radial magnetic field component is sheared out by the differential rotation to produce a time-dependent azimuthal field component. In this case, however, this is exactly compensated by an azimuthal velocity perturbation which creates azimuthal field in the opposite direction out of vertical field.

Further insight into the physical nature of this mode may be obtained by taking various limits. In the limit $k_{z} \rightarrow 0$, we have $\delta B_{R}=\delta \rho=\delta E=\delta \mathbf{F}=0$, and the only nontrivial condition comes from the radial momentum equation:

$$
-2 \Omega \rho \delta v_{\phi}+i k_{R} \delta p+i \frac{k_{R}}{4 \pi} B_{z} \delta B_{z}=0 .
$$


In other words, any random field of perturbations of $\delta v_{\phi}$ can be balanced by radial gradients of gas and/or magnetic pressure to produce a slightly different equilibrium.

In the opposite limit $k_{R} \rightarrow 0$, we have $\delta v_{\phi}=\delta B_{z}=\delta B_{R}=\delta F_{z}=0$, and

$$
\begin{gathered}
i k_{z}\left(\delta p+\frac{1}{3} \delta E\right)+g \delta \rho=0 \\
\delta F_{R}+\frac{i c}{3 \kappa_{e s} \rho} k_{R} \delta E=0 \\
F_{z} \frac{\delta \rho}{\rho}+\frac{i c}{3 \kappa_{e s} \rho} k_{z} \delta E=0
\end{gathered}
$$

In this limit, a random field of density perturbations is balanced by vertical gradients in gas and radiation pressure, again producing a slightly different equilibrium.

Finally, we note that if we neglect the vertical stratification of the equilibrium, then this zero mode has all perturbations vanishing except for the density. In this case a random field of density perturbations will merely be advected along with the equilibrium flow without producing additional net accelerations.

\section{REFERENCES}

Agol, E., \& Krolik, J. 1998, ApJ, 507, 304

Arons, J. 1992, ApJ, 388, 561

Balbus, S. A., \& Hawley, J. F. 1991, ApJ, 376, 214

Balbus, S. A., \& Hawley, J. F. 1998, Rev. Mod. Phys., 70, 1

Blaes, O. M., \& Balbus, S. A. 1994, ApJ, 421, 163

Gammie, C. F. 1998, MNRAS, 297, 929

Hawley, J. F., \& Balbus, S. A. 1992, ApJ, 400, 595

Krolik, J. H. 1998, ApJ, 498, L13

Lightman, A. P., \& Eardley, D. M. 1974, ApJ, 187, L1

Pietrini, P., \& Krolik, J. H. 2000, ApJ, 539, 216

Piran, T. 1978, ApJ, 221, 652 
Shakura, N. I., \& Sunyaev, R. A. 1973, A\&A, 24, 337

Shakura, N. I., \& Sunyaev, R. A. 1976, MNRAS, 175, 613

Stone, J. M., Mihalas, D., \& Norman, M. L. 1992, ApJ, 80, 819 


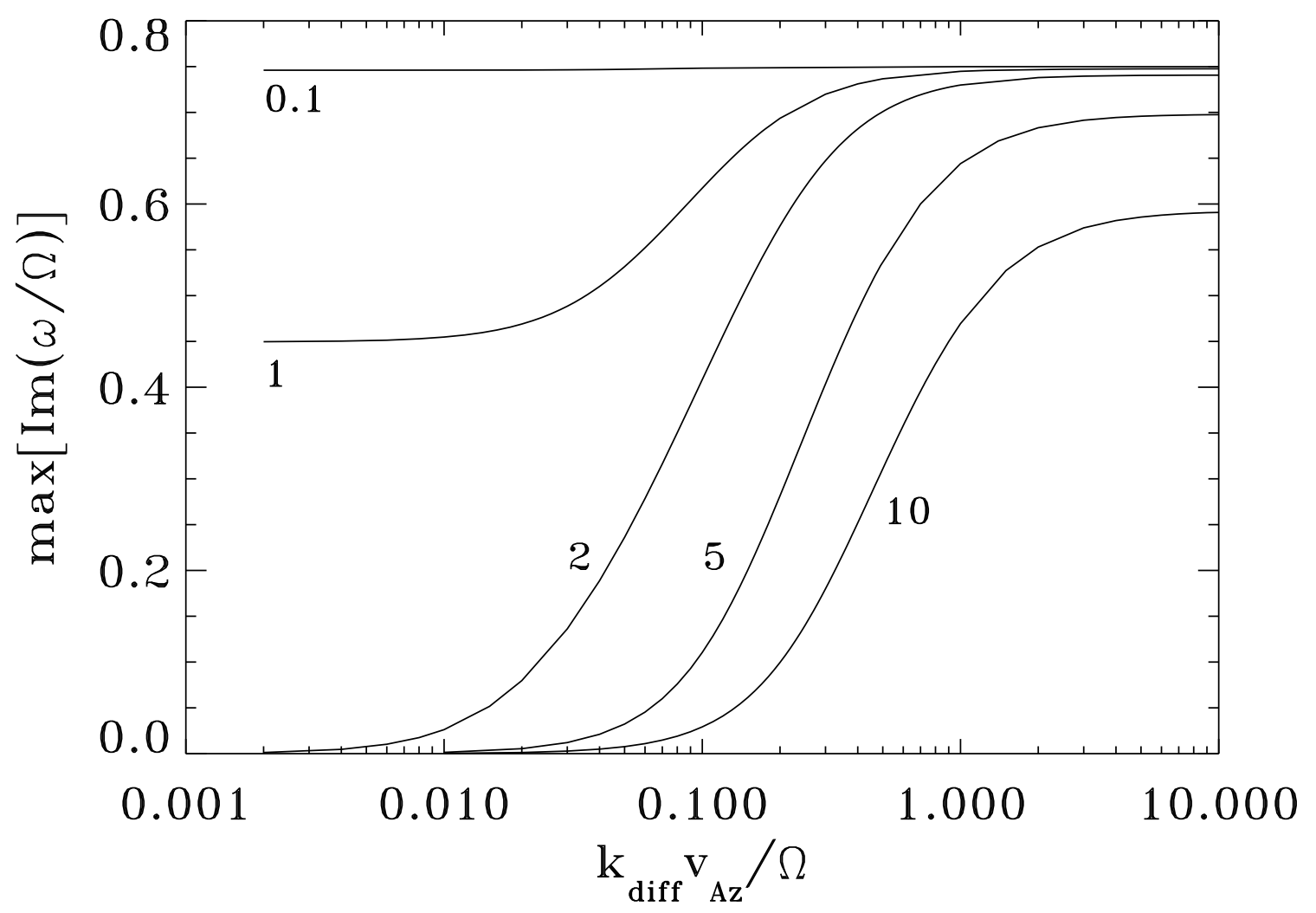

Fig. 1.- Maximum growth rate of the MRI in a medium with no stratification, as a function of azimuthal field strength and diffusion wavenumber. We have adopted a Keplerian rotation curve $(\kappa=\Omega)$ for these calculations, and have set $c_{\mathrm{g}}=0, c_{\mathrm{r}} / v_{\mathrm{A} z}=10$, and $k_{R}=0$. Each curve corresponds to different values of $v_{\mathrm{A} \phi} / v_{\mathrm{A} z}$, which are labelled. Note the substantial reduction in growth rate at modest azimuthal field strengths and low diffusion wavenumber. 

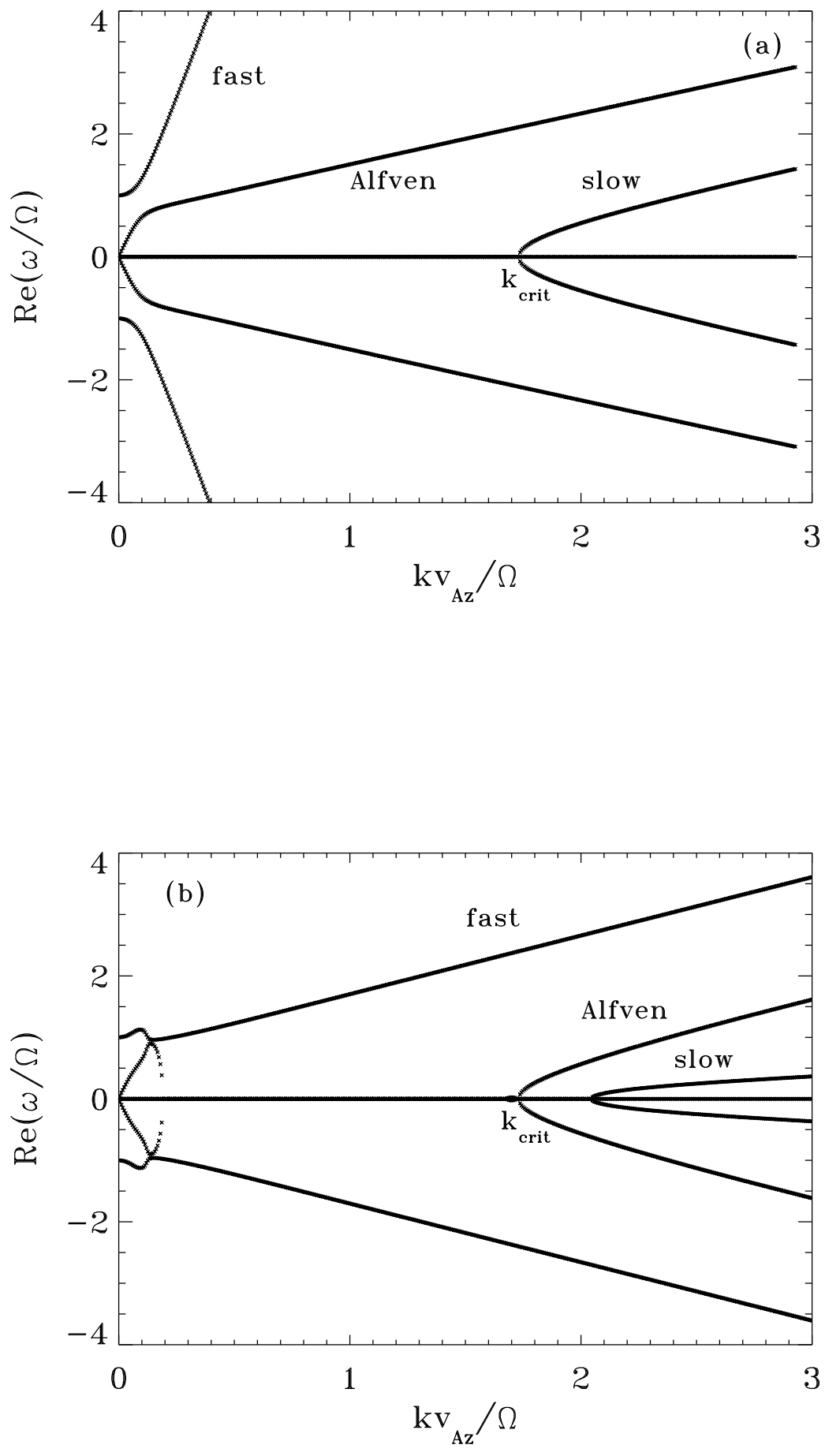


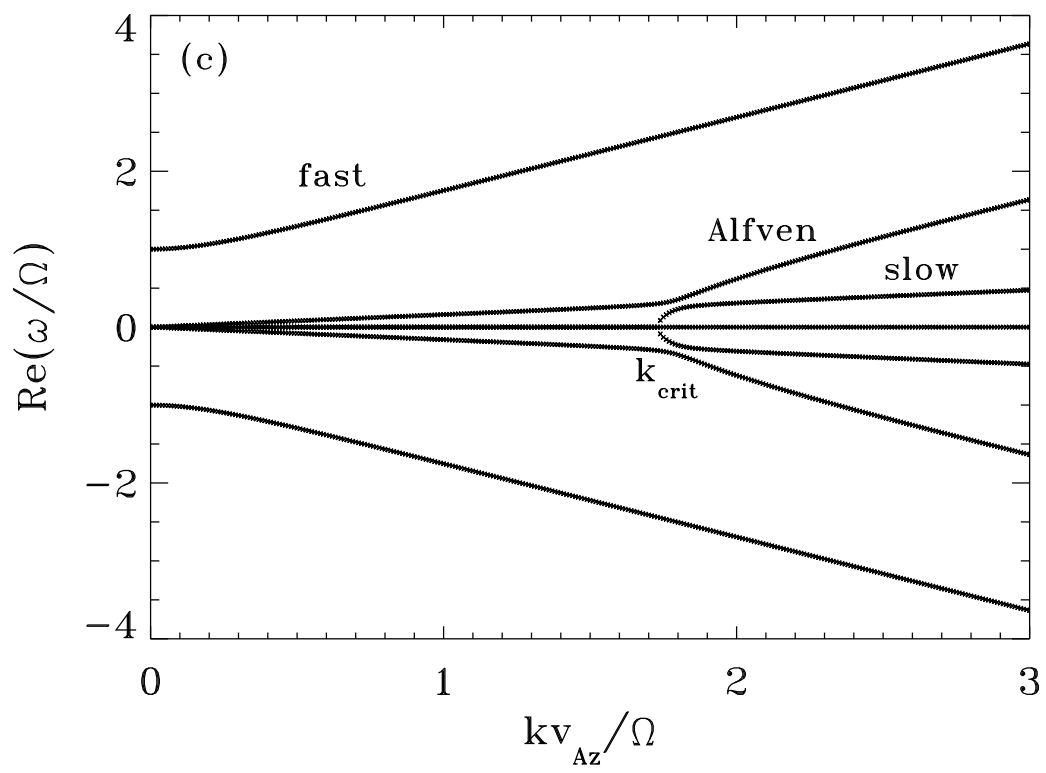

Fig. 2.- Real part of the mode frequencies in a medium with no stratification and a vertical magnetic field $\left(B_{\phi}=0\right)$, as a function of total wavenumber. We have assumed a Keplerian rotation curve and $k_{z} / k=0.8, c_{\mathrm{r}} / v_{\mathrm{A} z}=10$, and $c_{\mathrm{g}} / v_{\mathrm{A} z}=0.2$. Each figure corresponds to a different value of $k_{\mathrm{diff}} v_{\mathrm{A} z} / \kappa$ : (a) 10, (b) 0.1 , and (c) 0.01 . The critical wavenumber $k_{\text {crit }} \equiv\left[-R\left(d \Omega^{2} / d R\right)\right]^{1 / 2} / v_{\mathrm{A} z}$ above which the MRI is stabilized is indicated in each figure. 


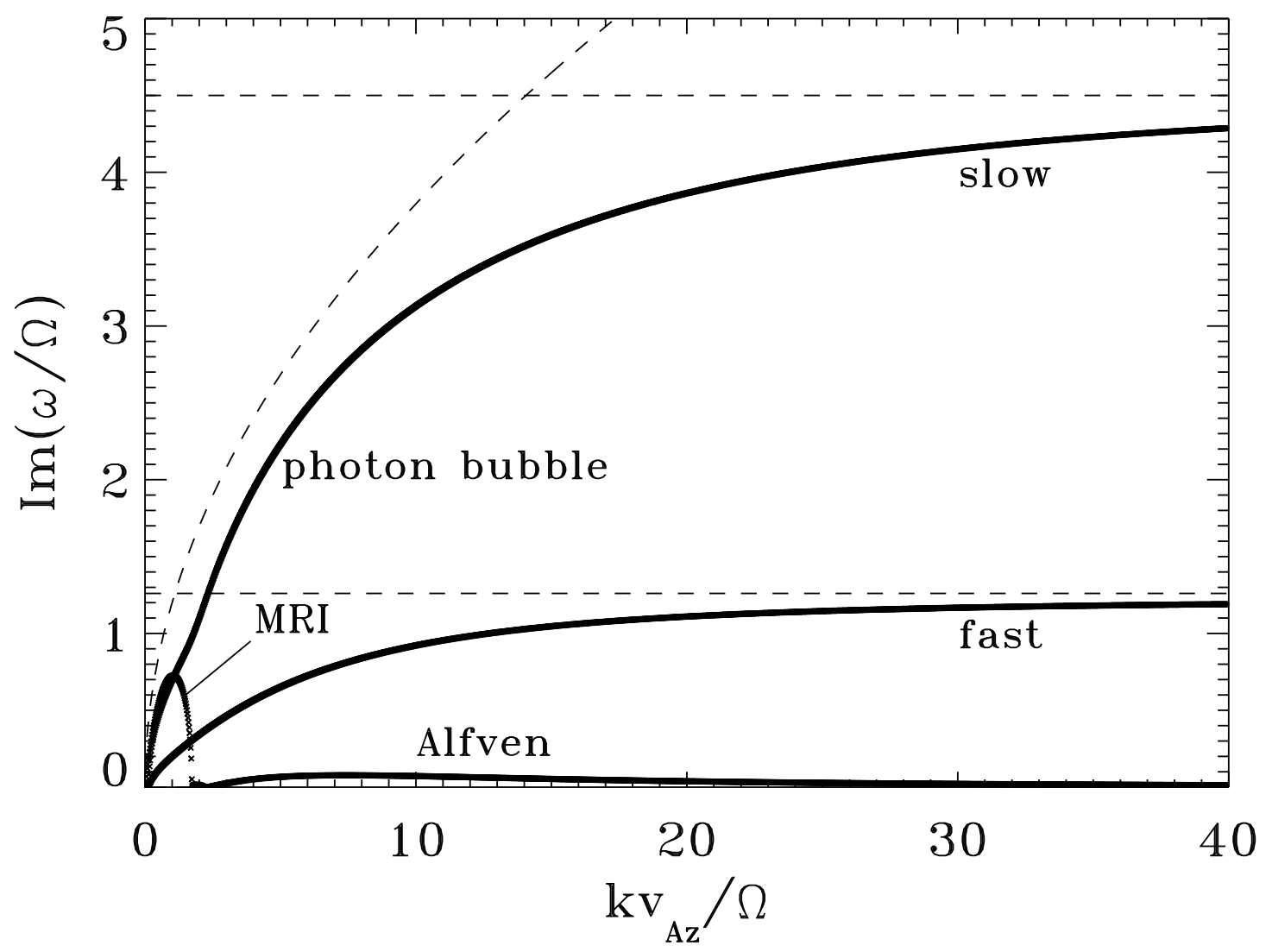

Fig. 3.- Imaginary part of the mode frequencies in a stratified medium with vertical magnetic field $\left(B_{\phi}=0\right)$ as a function of total wavenumber. The rotation curve is Keplerian and $k_{z} / k=0.8, c_{\mathrm{r}} / v_{\mathrm{A} z}=10, c_{\mathrm{g}} / v_{\mathrm{A} z}=0.4, k_{\mathrm{diff}} v_{\mathrm{A} z} / \kappa=0.1$, and $F_{z} /\left(3 \rho v_{\mathrm{A} z}^{3}\right)=1000$. The dashed curves show the analytic approximations (49), (53), and (54). [We have neglected the Brunt-Väisälä terms in the dispersion relation (46) and also set $g /\left(\kappa v_{\mathrm{A} z}\right)=10$ and $\left(g / \kappa^{2}\right) d \ln \rho / d z=-1$. However, these last two parameters do not significantly affect the results and could just as well have been set to zero. This is consistent with the WKB approximation.] 

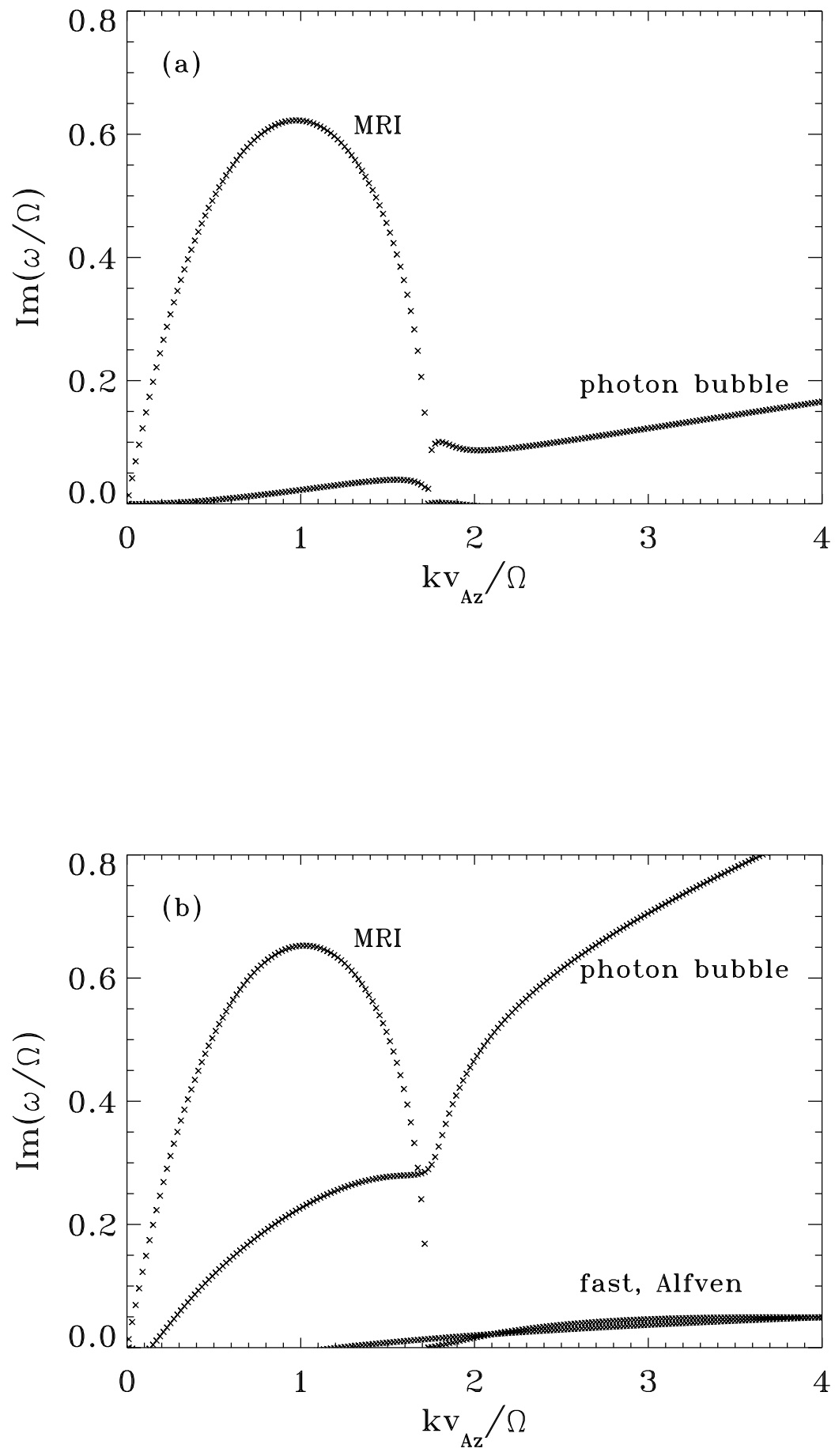


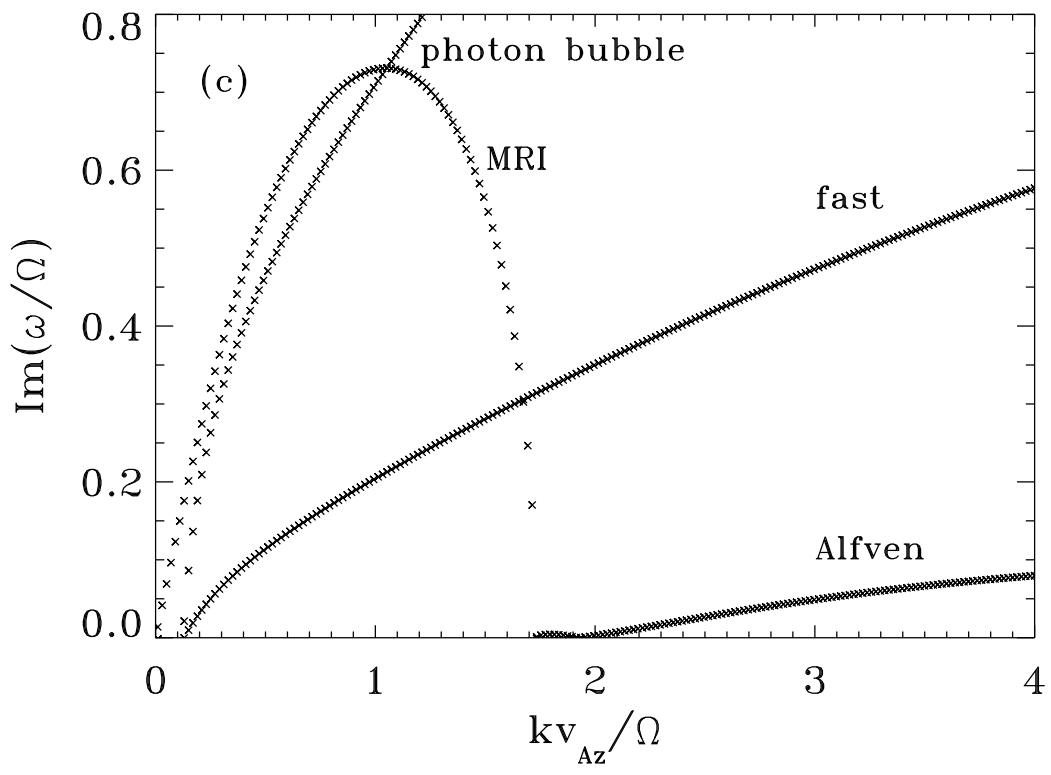

Fig. 4.- Imaginary part of the mode frequencies in a stratified medium with vertical magnetic field $\left(B_{\phi}=0\right)$ as a function of total wavenumber. The rotation curve is Keplerian and $k_{z} / k=0.8, c_{\mathrm{r}} / v_{\mathrm{A} z}=10, k_{\mathrm{diff}} v_{\mathrm{A} z} / \kappa=0.1$, and $F_{z} /\left(3 \rho v_{\mathrm{A} z}^{3}\right)=$ (a) 30, (b) 200, and (c) 1000. Gas pressure has been neglected and the vertical entropy gradient has been set to zero (i.e. $\left.N_{\mathrm{r}}=0\right)$.

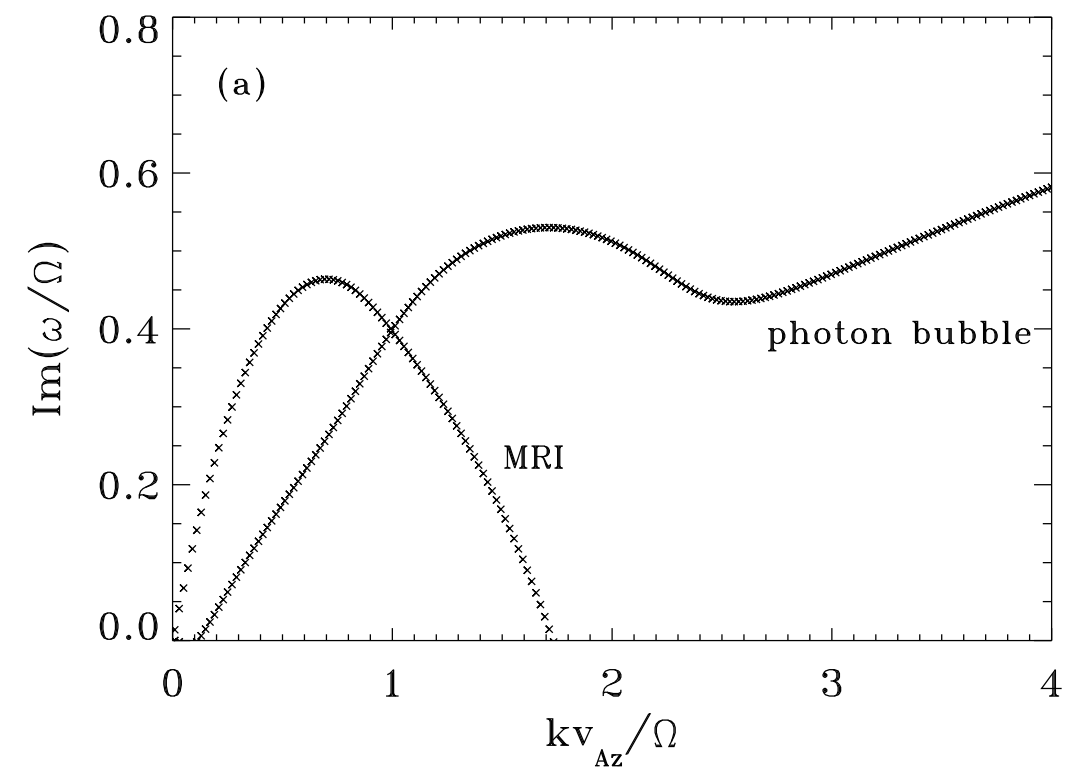




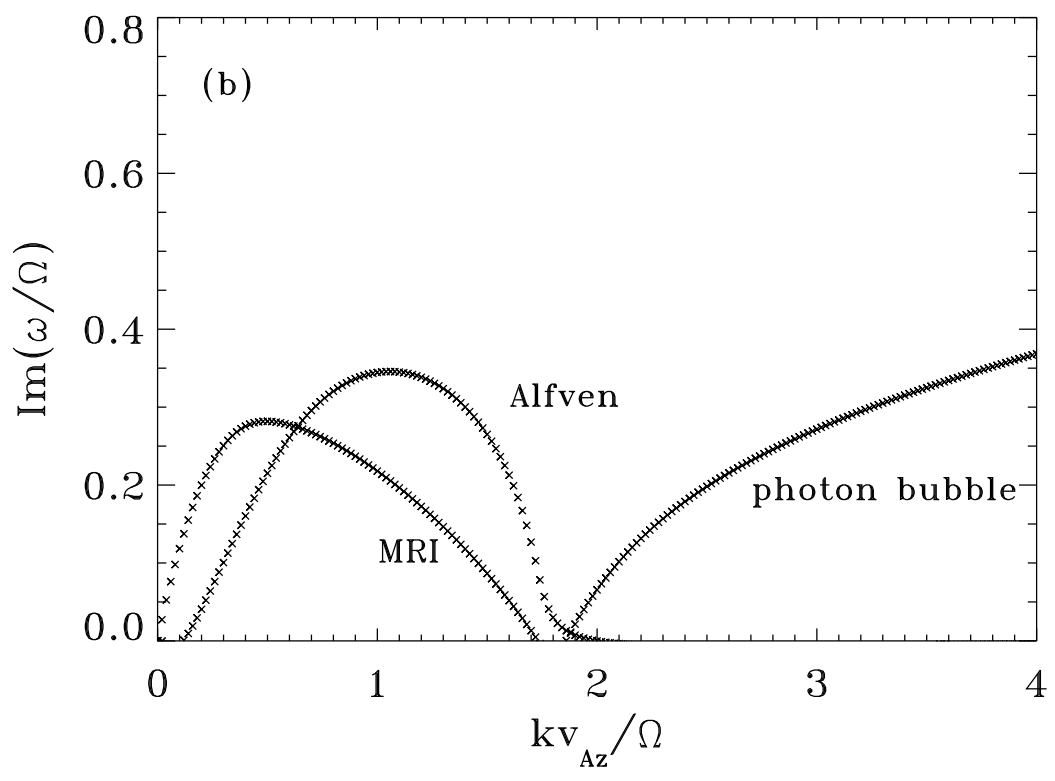

Fig. 5.- Same as figure 4(b) only with a nonvertical magnetic field: (a) $B_{\phi}=B_{z}$ and (b) $B_{\phi}=2 B_{z}$. 